PROCEEDINGS OF THE

AMERICAN MATHEMATICAL SOCIETY

Volume 135, Number 12, December 2007, Pages 3867-3873

S 0002-9939(07)08914-9

Article electronically published on August 29, 2007

\title{
BOUND ON THE NUMBER OF EIGENVALUES NEAR THE BOUNDARY OF THE PSEUDOSPECTRUM
}

\author{
MILDRED HAGER
}

(Communicated by Mikhail Shubin)

\begin{abstract}
We show an estimate of the number of eigenvalues in a neighbourhood of a finite part of the boundary of the semiclassical pseudospectrum of pseudodifferential non-selfadjoint operators in terms of a corresponding volume in phase space.
\end{abstract}

For non-selfadjoint operators, the norm of the resolvent can be large even far away from the spectrum. Pseudospectra are regions delimited by the level curves of this norm, and for semiclassical pseudodifferential operators one may study those corresponding to any negative power of the semiclassical parameter $h$. For an introduction as well as generalities we refer to [1, [2], 3], 14], 15].

In numerical calculations of the eigenvalues of some non-selfadjoint operator, an accumulation of these to the boundary of the semiclassical pseudospectrum has been observed ([16]), whereas the spectrum is known to be in its interior. This phenomenon has been a motivation for us to study in previous works the spectrum of perturbed semiclassical non-selfadjoint operators ([6], [7]), where the norm of the perturbation is exponentially small in $h$. We found a two-dimensional Weyl law in a domain inside the pseudospectrum. This implies that in contrast to what could be expected from the numerical observation not all the eigenvalues are close to the boundary. We prove here a complementary result, showing a bound on the number of eigenvalues of the (perturbed) operator near the boundary, thus next to a region where one can get better resolvent estimates. This observation has already been used for estimates of the number of resonances in a neighbourhood of the real axis, see [9], 10, 11, [8, and we refer to the first work for a historical overview as well as more references. The techniques we apply here are very similar.

\section{INTRODUCTION}

We will work with the same symbol classes and operators as in [7, and refer there and to [4 for a more detailed introduction. Let $P=p^{w}$ be the $h$-Weyl-quantization of some symbol $p$, acting in $L^{2}(\mathbb{R})$ :

$$
p^{w} u(x):=\frac{1}{2 \pi h} \iint e^{\frac{i}{h}(x-y) \xi} p\left(\frac{x+y}{2}, \xi\right) u(y) d y d \xi, 0<h \leq 1 .
$$

Received by the editors July 5, 2006 and, in revised form, August 18, 2006.

2000 Mathematics Subject Classification. Primary 34E10, 47G10, 47A75.

Key words and phrases. Pseudospectrum, perturbation, non-selfadjoint operators.

(C)2007 American Mathematical Society Reverts to public domain 28 years from publication 3867 
Assumption 1. Let $p \in S\left(\mathbb{R}^{2} ; m\right)$ be independent of $h$, where $m>1$ is an order function. Let $\Omega \Subset \mathbb{C}$ be an open connected set such that $p-z$ is elliptic at infinity uniformly in $\Omega$ and there is $z_{0} \in \Omega$ such that $p-z_{0}$ is elliptic.

Recall that $m \in C^{\infty}\left(\mathbb{R}^{2}\right)$ is an order function if there are $C>0$ and $N$ such that $0<m(\rho) \leq C\langle\rho-\eta\rangle^{N} m(\eta)$, for all $\rho, \eta \in \mathbb{R}^{2}$, and the corresponding symbol class $S\left(\mathbb{R}^{2} ; m\right)$ consists of those $p \in C^{\infty}\left(\mathbb{R}^{2}\right)$ with all derivatives being $O(m)$. Ellipticity in such a symbol class means that $|p|>\frac{1}{C} m$ for some $C>0$, and ellipticity at infinity means that this lower bound holds for all sufficiently large $\rho \in \mathbb{R}^{2}$. Assumption 1 guaranties that $P$ may be defined as a closed operator with dense domain $H(m)$ in $L^{2}$ (see [7]), having a purely discrete spectrum in $\Omega$ for $h>0$ small enough.

Let $\Sigma(p):=\overline{p\left(\mathbb{R}^{2}\right)}$. If $z \in \Omega \backslash \Sigma$, then $p-z$ is elliptic. We call $\Sigma(p)$ the semiclassical pseudospectrum of $p^{w}$. In fact, under additional assumptions there is a dense open subset of $\Sigma(p)$ where the resolvent norm is larger than any negative power of $h$, or exponentially large in the analytic case. We refer to [3] or 7] for these statements.

Here, we will give a bound on the number of eigenvalues of $p^{w}$ in a small strip around a finite part of $\partial \Sigma$.

Example 1. Let $p=\xi^{2}+i \xi+x^{2} \in S\left(\mathbb{R}^{2} ;\langle(x, \xi)\rangle^{2}\right)$,

$$
p^{w}=\left(h D_{x}\right)^{2}+h \partial_{x}+x^{2}
$$

(see, for example, [16]). We have $\Sigma=\left\{\operatorname{Re} z \geq(\operatorname{Im} z)^{2}\right\}$, whereas one can see using a formal conjugation by $e^{\frac{x}{2 h}}$ that $\operatorname{Spec}\left(p^{w}\right) \subset\left[\frac{1}{4}, \infty\right)$.

In order to use a covering argument later on, we need a geometric assumption on $\Sigma$. For $U \subset \mathbb{C}$, let $d(z, U)=\inf _{w \in U}|z-w|$, and for $r>0$ let $D(z, r):=\{w \in$ $\mathbb{C} ;|z-w|<r\}$. Also let $U^{c}=\mathbb{C} \backslash U$.

Assumption 2. For $p, \Omega$ as in Assumption 1, suppose there exists $C>0$ such that for all $\epsilon>0$ small enough and for all $z \in \partial \Sigma \cap \Omega$, there exists $z_{0} \in \Sigma^{c}$ such that $\left|z-z_{0}\right| \leq \epsilon$ and $d\left(z_{0}, \partial \Sigma\right) \geq \frac{\epsilon}{C}$.

If one assumes the exterior cone condition, i.e., that there exist $r_{0}>0, \theta_{0}>0$ such that for all $z \in \partial \Sigma \cap \Omega$, there exists $\theta(z)$ with

$$
\left\{z+r e^{i \theta}, 0<r \leq r_{0},|\theta-\theta(z)| \leq \theta_{0}\right\} \cap \Sigma=\emptyset,
$$

then Assumption 2 is satisfied. This condition is verified for Example1 with $\theta_{0}=\frac{\pi}{2}$ and every $r_{0}>0$.

Theorem 1. Let $p$ satisfy the assumptions 1 and 2 , Let $\widetilde{\Omega} \Subset \Omega$. Then there exist $C>0$ and $\epsilon_{0}>0$ such that if $0<\epsilon<\epsilon_{0}$, then there exists $h_{0}(\epsilon)>0$ such that if $W(\epsilon):=\{z \in \widetilde{\Omega} ; d(z, \partial \Sigma)<\epsilon\}$, then for $0<h \leq h_{0}$,

$$
\#\left(\operatorname{Spec}\left(p^{w}\right) \cap W(\epsilon)\right) \leq \frac{C}{h} \operatorname{vol}\left(p^{-1}(W(\epsilon)+D(0,14 \epsilon))\right) .
$$

Thus we obtain an estimate of the number of eigenvalues in a small neighbourhood of $\partial \Sigma$ in terms of a volume in phase space. This result is (via a Neumannseries argument) easily adapted to a small perturbation, which yields the following corollary. 
Corollary 2. Let $p$ and $\widetilde{\Omega}$ be as in Theorem 1. Let $Q$ be a bounded operator $L^{2} \rightarrow L^{2}$ and $H(m) \rightarrow H(m)$. Then there exist $\epsilon_{0}>0, D>0$ and $C>0$ such that if $0<\epsilon<\epsilon_{0}$, then there exists $h_{0}(\epsilon)>0$ such that if $W(\epsilon)$ is as in Theorem 1, then for $0<h \leq h_{0}$,

$$
\#\left(\operatorname{Spec}\left(p^{w}+\frac{\epsilon}{D} Q\right) \cap W(\epsilon)\right) \leq \frac{C}{h} \operatorname{vol}\left(p^{-1}(W(\epsilon)+D(0,14 \epsilon))\right) .
$$

Before proving Theorem 1 we investigate the volume in (4) and find an explicit upper bound under some non-degeneracy assumption.

\section{Explicit estimate in a NON-DEgenerate CASE}

For $f, g \in C^{\infty}\left(\mathbb{R}^{2}\right)$, the Poisson bracket is defined by

$$
\{f, g\}:=\left(\partial_{\xi} f\right) \partial_{x} g-\left(\partial_{x} f\right) \partial_{\xi} g .
$$

Suppose that $\{p,\{p, \bar{p}\}\}(\rho) \neq 0, \rho \in p^{-1}(\partial \Sigma \cap \Omega)$. Let us start by showing that this implies that for all $\Omega^{\prime} \Subset \Omega, p^{-1}\left(\partial \Sigma \cap \Omega^{\prime}\right)$ can be piecewise identified with isolated curves in $\mathbb{R}^{2}$.

Let $z^{*} \in \partial \Sigma \cap \Omega^{\prime}, \rho^{*} \in p^{-1}\left(z^{*}\right),\{p, \bar{p}\}\left(\rho^{*}\right)=0$, and concentrate on a neighbourhood of $\rho^{*} \cdot H_{\frac{1}{2 i}\{p, \bar{p}\}}$ is a real non-vanishing vector field tangent to the set $\{\rho ;\{p, \bar{p}\}(\rho)=0\}$. Therefore, for $t$ small enough,

$$
t \mapsto \rho^{*}(t):=\exp \left(t H_{\frac{1}{2 i}\{p, \bar{p}\}}\right)\left(\rho^{*}\right)
$$

is an oriented curve contained in $\{\rho ;\{p, \bar{p}\}(\rho)=0\}$, and $\frac{1}{2 i}\{p, \bar{p}\}$ is positive to the left of this curve, and negative to the right. $p\left(\rho^{*}(t)\right)$ is also an (oriented) curve, since

$$
\frac{d}{d t}\left(p\left(\rho^{*}(t)\right)\right)=\left(H_{\frac{1}{2 i}\{p, \bar{p}\}} p\right)\left(\rho^{*}(t)\right)=-\left\{p, \frac{1}{2 i}\{p, \bar{p}\}\right\}\left(\rho^{*}(t)\right) \neq 0
$$

by assumption.

Furthermore, $d \operatorname{Im} p \wedge d \operatorname{Re} p=\frac{1}{2 i}\{p, \bar{p}\} d \xi \wedge d x$, so $\rho \rightarrow p(\rho)$ preserves the orientation to the left of $\rho^{*}(t)$ and reverses it to the right. Thus $p(\rho)$ is on the left of the curve $p\left(\rho^{*}(t)\right), \rho \in \operatorname{neigh}\left(\rho^{*}(t)\right)$, and $\Sigma$ is the union of such regions. Hence $\partial \Sigma$ is given locally by the image under $p$ of some curve of the form (77). $p^{-1}\left(\partial \Sigma \cap \Omega^{\prime}\right)$ can be locally identified with curves of the type (7), and $p^{-1}\left(z^{*}\right)=\left\{\rho_{1}^{*}, \ldots, \rho_{n}^{*}\right\}$. Let $\left(H_{\frac{1}{2 i}\{p, \bar{p}\}} p\right)\left(\rho_{j}^{*}\right):=r_{j}^{*} e^{i \theta_{j}^{*}}$.

Assumption 3. There is an $L \in(0, \pi)$ such that for all $z^{*} \in \partial \Sigma \cap \Omega$, there is an interval $I_{z^{*}} \subset \mathbb{R}$ of length $L$ and a multi-index $\alpha \in \mathbb{Z}^{n}$ such that $\left\{\theta_{1}^{*}+\alpha_{1} 2 \pi, \ldots, \theta_{n}^{*}+\right.$ $\left.\alpha_{n} 2 \pi\right\} \subset I_{z^{*}}$.

The union of the regions to the left of the curves $p\left(\rho_{j}^{*}(t)\right)$ leave a conical region in $\mathbb{C}$ uncovered, with opening angle $\theta_{0}=\pi-L>0$, which is the exterior cone condition; thus Assumption 3 implies Assumption 2.

Corollary 3. Let $p$ satisfy Assumption 1. If moreover

$$
\{p,\{p, \bar{p}\}\}(\rho) \neq 0, \rho \in p^{-1}(\Omega \cap \partial \Sigma),
$$

and Assumption 3 is fulfilled, then there exist $C>0$ and $\epsilon_{0}>0$ such that if $0<\epsilon<\epsilon_{0}$, then there exists $h_{0}(\epsilon)>0$ such that if $W(\epsilon)$ is as in Theorem 1, then for $0<h \leq h_{0}$,

$$
\#\left(\operatorname{Spec}\left(p^{w}\right) \cap W(\epsilon)\right) \leq C \frac{\sqrt{\epsilon}}{h}
$$


Thus in this case we obtain an explicit bound on the number of eigenvalues in some neighbourhood of the boundary, and it holds in particular for Example 1 .

Proof of Corollary 3 , For $\epsilon$ small enough, $(\widetilde{\Omega}+D(0,14 \epsilon))=\Omega^{\prime} \Subset \Omega$, and the preceeding analysis applies. Let $z^{*} \in \partial \Sigma \cap \Omega^{\prime}$, and let $\rho_{j}^{*} \in p^{-1}\left(z^{*}\right)$. Assume without loss of generality that if $\rho \in p^{-1}(\Omega \cap \partial \Sigma)$,

$$
\{\operatorname{Re} p,\{p, \bar{p}\}\}(\rho) \neq 0 \text {. }
$$

This means that $H_{\operatorname{Re} p}$ and $H_{\frac{1}{2 i}\{p, \bar{p}\}}$ are linearly independent at $\rho_{j}^{*}$. Thus any $\rho$ in a small neighbourhood of $\rho_{j}^{*}$ may be written as

$$
\rho=\exp \left(s H_{\operatorname{Re} p}\right) \exp \left(t H_{\frac{1}{2 i}\{p, \bar{p}\}}\right)\left(\rho_{j}^{*}\right)=\exp \left(s H_{\operatorname{Re} p}\right)\left(\rho_{j}^{*}(t)\right),
$$

where $t$ parametrizes (as in (7)) the position along the (possible) preimage of the boundary. We have

$$
p\left(\exp \left(s H_{\operatorname{Re} p}\right)\left(\rho_{j}^{*}(t)\right)\right)=p\left(\rho_{j}^{*}(t)\right)+\frac{i s^{2}}{2}\{\operatorname{Re} p,\{\operatorname{Re} p, \operatorname{Im} p\}\}\left(\rho_{j}^{*}(t)\right)+O_{t}\left(s^{3}\right) .
$$

Using $\{\operatorname{Re} p,\{\operatorname{Re} p, \operatorname{Im} p\}\}\left(\rho_{j}^{*}(t)\right) \neq 0$, we obtain

$$
\begin{aligned}
& \operatorname{vol}\left(p^{-1}\left((W(\epsilon)+D(0,14 \epsilon)) \cap \operatorname{neigh}\left(z^{*}\right)\right)\right) \\
& \leq \sum_{j} \operatorname{vol}\left(\left\{\exp \left(s H_{\operatorname{Re} p}\right) \exp \left(t H_{\frac{1}{2 i}\{p, \bar{p}\}}\right)\left(\rho_{j}^{*}\right) ; t \in\left(t_{1}, t_{2}\right), s^{2}<C \epsilon\right\}\right) \\
& =O(\sqrt{\epsilon}) .
\end{aligned}
$$

We can cover $W(\epsilon)+D(0,14 \epsilon)$ by a finite number of such neighbourhoods; hence the same estimate holds for $W(\epsilon)+D(0,14 \epsilon)$.

\section{Proof of Theorem 1}

We first establish the estimate in a small disc.

Lemma 4. Let $p$ satisfy the Assumptions 1 and 2 , Let $\widetilde{\Omega} \Subset \Omega$. Then there exist $C^{\prime}>0$ and $\epsilon_{0}>0$ such that if $0<\epsilon<\epsilon_{0}$, and $z_{0} \in \Omega \backslash \Sigma, \frac{\epsilon}{C} \leq d\left(z_{0}, \Sigma\right) \leq \epsilon$, $d\left(z_{0}, \overline{\widetilde{\Omega}}\right) \leq 2 \epsilon$, there exists $h_{0}(\epsilon)>0$ such that if $0<h \leq h_{0}$, then

$$
\#\left(\operatorname{Spec}\left(p^{w}\right) \cap D\left(z_{0}, \epsilon\right)\right) \leq \frac{C^{\prime}}{h} \operatorname{vol}\left(p^{-1}\left(D\left(z_{0}, 3 \epsilon\right)\right)\right) .
$$

Before proving the lemma, we finish the proof of Theorem 1 using a covering argument.

Proof of Theorem 1. Let $\left\{D\left(z_{k}, \epsilon\right), k \in K\right\}$ be a maximal family of disjoint open discs such that $z_{k} \in \Omega \backslash \Sigma$ are as in Lemma 4, Then

$$
W(\epsilon) \subset \bigcup_{k} D\left(z_{k}, 4 \epsilon\right),
$$

which we will prove by a contradiction argument. Let us assume there is $z \in W(\epsilon)$ with $\left|z-z_{k}\right| \geq 4 \epsilon, \forall k$. Let $z^{*} \in \partial \Sigma \cap \Omega$ fulfill $\left|z-z^{*}\right| \leq \epsilon$, and let $\tilde{z} \in \Omega \backslash \Sigma$ be such that

$$
\left|\tilde{z}-z^{*}\right| \leq \epsilon, d(\tilde{z}, \partial \Sigma) \geq \frac{\epsilon}{C}
$$

Then $\tilde{z}$ is as in Lemma 4, and

$$
\left|\tilde{z}-z_{k}\right| \geq\left|z-z_{k}\right|-\left|z^{*}-z\right|-\left|\tilde{z}-z^{*}\right| \geq 2 \epsilon, \forall k
$$


Hence $\left\{D\left(z_{k}, \epsilon\right), k \in K\right\} \cup\{D(\tilde{z}, \epsilon)\}$ is a family of disjoint discs as above, which contradicts the maximality of $\left\{D\left(z_{k}, \epsilon\right), k \in K\right\}$.

Using Lemma 4, we obtain

$$
\left.\#\left(\operatorname{Spec}\left(p^{w}\right) \cap W(\epsilon)\right) \leq \frac{C}{h} \sum_{k} \operatorname{vol}\left(p^{-1}\left(D\left(z_{k}, 12 \epsilon\right)\right)\right)\right) .
$$

Since $\left|z_{k}-z_{j}\right| \geq 2 \epsilon, \forall k \neq j$, there exists $C>0$ such that every $z \in \bigcup_{k} D\left(z_{k}, 12 \epsilon\right)$ is contained in at most $C$ discs. Therefore, using also $d\left(z_{k}, \Sigma\right) \geq \frac{\epsilon}{C}$,

$$
\sum_{k} \operatorname{vol}\left(p^{-1}\left(D\left(z_{k}, 12 \epsilon\right)\right)\right) \leq C \operatorname{vol}\left(p^{-1}(W(\epsilon)+D(0,14 \epsilon))\right) .
$$

Proof of Lemma 4. Let $z \in D\left(z_{0}, 2 \epsilon\right)$. We construct an auxiliary operator having the same spectrum as $p^{w}$ in $D\left(z_{0}, 2 \epsilon\right)$, whose determinant is well defined.

Since $p$ is elliptic at infinity, $p^{-1}\left(D\left(z_{0}, \frac{5}{2} \epsilon\right) \cap \Sigma\right)$ is bounded. Let

$$
k_{\epsilon}(x, \xi)=2 \epsilon \frac{p-z_{0}}{\left|p-z_{0}\right|} \chi_{\epsilon}(x, \xi),
$$

$\chi_{\epsilon} \in C_{c}^{\infty}\left(p^{-1}\left(D\left(z_{0}, 3 \epsilon\right) \cap \Sigma\right) ;[0,1]\right)$ be such that $\chi_{\epsilon}=1$ on $p^{-1}\left(D\left(z_{0}, \frac{5}{2} \epsilon\right) \cap \Sigma\right)$, and

$$
\left\|\partial^{\alpha} \chi_{\epsilon}\right\|_{L^{\infty}}=O_{\alpha}(1) \epsilon^{-|\alpha|} .
$$

Then

$$
\begin{aligned}
\left|p+k_{\epsilon}-z\right| & \geq\left|p+k_{\epsilon}-z_{0}\right|-\left|z-z_{0}\right| \geq\left|p-z_{0}\right|+\left|k_{\epsilon}\right|-2 \epsilon \\
& \geq\left\{\begin{array}{l}
\frac{\epsilon}{2}, \quad\left|p-z_{0}\right| \geq \frac{5}{2} \epsilon \\
\frac{\epsilon}{C}+2 \epsilon-2 \epsilon, \quad\left|p-z_{0}\right| \leq \frac{5}{2} \epsilon
\end{array}\right.
\end{aligned}
$$

where we have used that $d\left(z_{0}, \Sigma\right) \geq \frac{\epsilon}{C}$. Moreover, using the above estimate in a compact set, $\left|p+k_{\epsilon}-z\right| \geq \frac{\epsilon}{C} m$. Therefore, for $\epsilon>0$ fixed, $p+k_{\epsilon}-z \in S\left(\mathbb{R}^{2} ; m\right)$ is elliptic and

$$
P_{\epsilon}:=\left(p+k_{\epsilon}-z\right)^{w}: H(m) \rightarrow L^{2}
$$

is invertible if $h$ is small enough depending on $\epsilon$ (see [7]).

Using the semiclassical sharp Gårding inequality (for $P^{*} P$, see for example [4]), we obtain that for $z \in D\left(z_{0}, 2 \epsilon\right)$ :

$$
\left\|\left(P_{\epsilon}-z\right)^{-1}\right\| \leq \sup \left(\left|p+k_{\epsilon}-z\right|^{-1}\right)+C(\epsilon) h \leq \frac{\tilde{C}}{\epsilon}
$$

if $h$ is small enough with respect to $\epsilon$. Furthermore

$$
p^{w}-z=\left(1-k_{\epsilon}^{w}\left(P_{\epsilon}-z\right)^{-1}\right)\left(P_{\epsilon}-z\right),
$$

and for $z \in D\left(z_{0}, 2 \epsilon\right), p^{w}-z$ is invertible if and only if $1-k_{\epsilon}^{w}\left(P_{\epsilon}-z\right)^{-1}$ is. Thus, the spectrum of $p^{w}$ in $D\left(z_{0}, 2 \epsilon\right)$ is determined by the zeros of the corresponding determinant.

We first prove that the trace class norm of $k_{\epsilon}^{w}$ obeys

$$
\left\|k_{\epsilon}^{w}\right\|_{t r} \leq \frac{\tilde{C} V(3 \epsilon) \epsilon}{h}
$$


where $V(\epsilon):=\operatorname{vol}\left(p^{-1}\left(D\left(z_{0}, \epsilon\right)\right)\right)$. In fact, for $a \in C_{b}^{\infty}\left(\mathbb{R}^{2}\right)$,

$$
\left\|O p^{w}(a)\right\|_{t r} \leq C \sum_{|\alpha| \leq 3}\left\|\partial_{(x, \xi)}^{\alpha} a\right\|_{L^{1}},
$$

where $O p^{w}(a)$ denotes the Weyl-quantization of $a$ for $h=1$ (see for example 4, theorem 9.4). $k_{\epsilon}^{w}$ is unitarily equivalent to $O p^{w}\left(k_{\epsilon, h}\right)$, where $k_{\epsilon, h}(x, \xi)=k_{\epsilon}(\sqrt{h} x, \sqrt{h} \xi)$. Therefore

$$
\left\|k_{\epsilon}^{w}\right\|_{t r} \leq C \sum_{|\alpha| \leq 3}\left\|\partial_{(x, \xi)}^{\alpha} k_{\epsilon, h}\right\|_{L^{1}} \leq C \sum_{|\alpha| \leq 3} h^{\frac{|\alpha|}{2}-1}\left\|\partial_{(x, \xi)}^{\alpha} k_{\epsilon}\right\|_{L^{1}} .
$$

Since $\left\|\partial_{(x, \xi)}^{\alpha} k_{\epsilon}\right\|_{L^{1}} \leq C V(3 \epsilon) \epsilon^{1-|\alpha|}$, this yields (24) if $h \leq h_{0}(\epsilon)$.

Thus $k_{\epsilon}^{w}\left(P_{\epsilon}-z\right)^{-1}$ is of trace class, and (see [4])

$$
\left\|k_{\epsilon}^{w}\left(P_{\epsilon}-z\right)^{-1}\right\|_{t r} \leq\left\|\left(P_{\epsilon}-z\right)^{-1}\right\|\left\|k_{\epsilon}^{w}\right\|_{t r} \leq \tilde{C} \frac{V(3 \epsilon)}{h} .
$$

Following [5], we can introduce

$$
D(z):=\operatorname{det}\left(1-k_{\epsilon}^{w}\left(P_{\epsilon}-z\right)^{-1}\right)
$$

and

$$
|D(z)| \leq \exp \left(\left\|k_{\epsilon}^{w}\left(P_{\epsilon}-z\right)^{-1}\right\|_{t r}\right) \leq e^{\frac{\tilde{C} V(3 \epsilon)}{h}} .
$$

The eigenvalues of $p^{w}$ are given by the zeros of $D(z)$, the number of which we will estimate using Jensen's formula (see, for example, [13]). We need a lower bound on $\left|D\left(z_{0}\right)\right| \cdot p^{w}-z_{0}$ is invertible, and

$$
\left\|\left(p^{w}-z_{0}\right)^{-1}\right\|_{L^{2} \rightarrow H(m)} \leq \frac{C^{\prime \prime}}{\epsilon} .
$$

Let $K_{\epsilon}:=k_{\epsilon}^{w}\left(P_{\epsilon}-z_{0}\right)^{-1},\left\|K_{\epsilon}\right\|=O(1): L^{2} \rightarrow L^{2}$,

$$
\left\|K_{\epsilon}\right\|_{t r} \leq\left\|\left(P_{\epsilon}-z_{0}\right)^{-1}\right\|\left\|k_{\epsilon}^{w}\right\|_{t r} \leq \frac{C^{\prime} V(3 \epsilon)}{h} .
$$

From (23), we obtain that $1-K_{\epsilon}$ is invertible and that

$$
\left(1-K_{\epsilon}\right)^{-1}=\left(P_{\epsilon}-z_{0}\right)\left(p^{w}-z_{0}\right)^{-1}=O(1): L^{2} \rightarrow L^{2} .
$$

Using $\left(1-K_{\epsilon}\right)^{-1}=1+K_{\epsilon}+\left(1-K_{\epsilon}\right)^{-1} K_{\epsilon}^{2}$, we get

$$
\left|D\left(z_{0}\right)\right|^{-1} \leq \exp \left(\left\|K_{\epsilon}+\left(1-K_{\epsilon}\right)^{-1} K_{\epsilon}^{2}\right\|_{t r}\right) .
$$

Since

$$
\left\|K_{\epsilon}+\left(1-K_{\epsilon}\right)^{-1} K_{\epsilon}^{2}\right\|_{t r} \leq\left(1+\left\|\left(1-K_{\epsilon}\right)^{-1}\right\|\left\|K_{\epsilon}\right\|\right)\left\|K_{\epsilon}\right\|_{t r} \leq C\left\|K_{\epsilon}\right\|_{t r},
$$

we obtain

$$
\left|D\left(z_{0}\right)\right| \geq e^{-\frac{C^{\prime} V(3 \epsilon)}{h}} .
$$

Applying Jensen's formula to $D(z)$ in $D\left(z_{0}, 2 \epsilon\right)$, together with (29), (35) as for instance in [11, 12] finishes the proof.

\section{ACKNOWLEDGMENTS}

This work is part of the thesis of the author under the supervision of J. Sjöstrand, whom she would like to thank here for very inspiring meetings. 


\section{REFERENCES}

[1] E.B. Davies, Semiclassical states for Non-Self-Adjoint Schrödinger Operators, Commun. Math. Phys. 200 (1999), 35-41 MR.1671904 (99m:34197)

[2] E.B. Davies, Pseudospectra of differential operators, J. Operator Theory 43 (2000), 243-262 MR $1753408(2001 \mathrm{~b}: 47034)$

[3] N. Dencker, J. Sjöstrand, M. Zworski, Pseudospectra of semiclassical (pseudo-) differential operators, Comm. Pure Appl. Math. 57 (2004), 384-415 MR2020109 (2004k:35432)

[4] M. Dimassi, J. Sjöstrand, Spectral Asymptotics in the Semi-Classical Limit, London Math. Soc., Lecture Note Series 268, Cambridge University Press (1999) MR.1735654(2001b:35237)

[5] I.C. Gohberg, M.G. Krein, Introduction to the theory of linear nonselfadjoint operators, Translations of Mathematical Monographs 18, Amer. Math. Soc. (1969) MR0246142 $(39: 7447)$

[6] M. Hager, Instabilité spectrale semiclassique d'opérateurs non-autoadjoints I: un exemple, Annales de la faculté des sciences de Toulouse no.2, volume 15 (2006), 195-232 MR.2244217

[7] M. Hager, Instabilité spectrale semiclassique d'opérateurs non-autoadjoints II, Annales Henri Poincaré 7 (2006), 1035-1064. MR2267057

[8] B. Helffer, J. Sjöstrand, Résonances en limite semi-classique, Bulletin de la Soc. Math. France (1986)

[9] J. Sjöstrand, Geometric bounds on the density of resonances for semiclassical problems, Duke Mathematical Journal 60 (1990), 1-57 MR:1047116 (91e:35166)

[10] J. Sjöstrand, M. Zworski, Complex scaling and the distribution of scattering poles, Jour. Amer. Math. Soc. 4 (1991), 729-769 MR1115789 (92g:35166)

[11] J. Sjöstrand, Lectures on resonances, http://daphne.math.polytechnique.fr/ sjoestrand/

[12] J. Sjöstrand, Resonances for bottles and trace formulae, Math. Nachr. 221 (2001), 95-149. MR.1806367 (2001k:58063)

[13] E.C. Titchmarsh, The theory of functions, Oxford University Press (1939)

[14] L.N. Trefethen, Pseudospectra of linear operators, SIAM Rev. 39 (1997), 383-406 MR.1469941 (98i:47004)

[15] M. Zworski, A remark on a paper of E.B. Davies, Proceedings of the AMS 129 (1999), 29552957 MR 1840099 (2002e:35015)

[16] M. Zworski, Numerical linear algebra and solvability of partial differential equations, Comm. Math. Phys. 229 (2002), 293-307 MR1923176 (2003i:35008)

CMls, Ecole polytechnique, 91128 Palaiseau Cédex, France, UMR 7640

E-mail address: mildred.hager@math.polytechnique.fr 